\title{
DEVELOPMENT OF THE MAPPING FUNCTION AT AN ANALYTIC CORNER
}

\author{
R. Sherman Lehman
}

1. Introduction. In this paper we shall apply some theorems proved in [3] to study the following problem in conformal mapping. Let $D$ be a domain of the complex plane, the boundary of which in the neighborhood of the origin consists of portions of two analytic curves $\Gamma_{1}$ and $\Gamma_{2}$. Suppose $\Gamma_{1}$ and $\Gamma_{2}$ meet at the origin and form a corner with opening $\pi \alpha>0$, and suppose the origin is a regular point of both curves. Let $F(z)$ be a function which maps conformally the upper half plane $\Im z>0$ onto the domain $D$, and suppose that $F(0)=0$. How does the mapping function $F(z)$ behave in the neighborhood of the origin?

A partial answer to this question is given by a theorem stated by Lichtenstein [5]. Let $F^{-1}(z)$ be the inverse function which maps $D$ onto the upper half plane. Then Lichtenstein stated that for $z$ in the neighborhood of the origin

$$
d F^{-1}(z)=z^{1 / \alpha-1} \varphi(z)
$$

where $\varphi(z)$ is a continuous function with $\varphi(0) \neq 0 .{ }^{1}$ This same result can, however, be obtained with much weaker requirements on the boundary curve as has been shown by the work of Kellogg [2] and Warschawski [6].

In the case $\alpha=1$ where the curves $\Gamma_{1}$ and $\Gamma_{2}$ meet at a straight angle Lewy [4] has proved a much stronger result-that $F(z)$ has an asymptotic expansion in powers of $z$ and $\log z$. The method used in this paper is a generalization of that used by Lewy. We find that for all $\alpha>0$ the function $F(z)$ has an asymptotic expansion in the neighborhood of the origin. If $\alpha$ is irrational then the expansion is in integral powers of $z$, and $z^{\alpha}$. If $\alpha$ is rational then the expansion is in integral powers of $z, z^{\alpha}$, and $\log z$.

2. Notation. First let us make clear what type of asymptotic expansions we will be considering. Let $\chi_{n}(z),(n=0,1,2, \cdots)$ be a sequence of functions such that $\chi_{n+1}(z) / \chi_{n}(z) \rightarrow 0$ as $z \rightarrow 0$ in the sector

Received September 11, 1956. This work was sponsored by the Office of Naval Research and the National Science Foundation. It appeared in report form as Tech. Report No. 21, Contract Nonr 225(11), March 1954. The author wishes to acknowledge the guidance of Professor Hans Lewy.

${ }^{1}$ Lichtenstein proved this result only in the case of irrational $\alpha$. The complete theorem has been proved recently by Warschawski [ 7$]$. 
$\theta_{1} \leqq \arg z \leqq \theta_{2}$. A series $\sum_{n=0}^{\infty} A_{n} \chi_{n}(z)$ is called an asymptotic expansion for $f(z)$ valid in the sector $\theta_{1} \leqq \arg z \leqq \theta_{2}$, and we write

$$
f(z) \sim \sum_{n=0}^{\infty} A_{n} \chi_{n}(z)
$$

if for every integer $N \geq 0$

$$
f(z)=\sum_{n=0}^{N} A_{n} \chi_{n}(z)+o\left(\chi_{N}(z)\right)
$$

as $z \rightarrow 0, \theta_{1} \leqq \arg z \leqq \theta_{2}$.

Clearly, in a sector $\theta_{1} \leqq \arg z \leqq \theta_{2}$ a function $f(z)$ cannot have more than one asymptotic expansion in terms of such a sequence of functions $\chi_{n}(z)$.

We shall sometimes be concerned with asymptotic expansions which are valid in every finite sector on the logarithmic Riemann surface with the origin as branch point. By this we mean that the limits hold for $z \rightarrow 0$ in any finite sector $\theta_{1} \leqq \arg z \leqq \theta_{2}$ where $\theta_{1}$ and $\theta_{2}$ are arbitrary constants. Otherwise expressed, we consider any sequence $z_{1}, z_{2}, z_{3}, \cdots$ such that there exist constants $\theta_{1}$ and $\theta_{2}$ for which

$$
\theta_{1} \leqq \arg z_{n} \leqq \theta_{2} \quad(n=1,2,3, \cdots)
$$

and

$$
\lim _{n \rightarrow \infty}\left|z_{n}\right|=0
$$

Thus we exclude any sequence for which $\lim \sup \left|\arg z_{n}\right|=\infty$.

Throughout this paper we will use the letter $c$ to denote a typical coefficient in a series when the exact value of the coefficient is not important in the discussion. For example, instead of writing $\sum_{n=0}^{\infty} c_{m n} z^{n}$, we may write simply $\sum_{n=0}^{\infty} c z^{n}$. Thus we avoid a multiplicity of subscripts.

3. Principal results. Let $F(z)$ be the mapping function which maps the upper half plane onto the domain $D$, and let $\Gamma_{1}$ be the image of a portion of the negative real axis and $\Gamma_{2}$ the image of a portion of the positive real axis. We shall prove the following theorem.

THEOREM 1. If $\alpha>0$ is irrational, then for $z \rightarrow 0$ in any finite sector

$$
F(z) \sim \sum A_{k l} z^{k+l \alpha}
$$

where $k$ and $l$ run over integers, $k \geq 0, l \geq 1$; and the coefficient $A_{01} \neq 0$. If $\alpha=p / q>0$, a fraction reduced to lowest terms, then for $z \rightarrow 0$ in any finite sector 


$$
F(z) \sim \sum A_{k l m} z^{k+l \alpha}(\log z)^{m}
$$

where $k, l$, and $m$ run over integers for which

$$
k \geqq 0, \quad 1 \leqq l \leqq q, \quad 0 \leqq m \leqq k / p ;
$$

and the coefficient $A_{010} \neq 0$.

In this theorem the terms in the series are supposed to be arranged in an order such that a term of the form $z^{k+l \alpha}(\log z)^{m}$ precedes one of the form $z^{k^{\prime}+l^{\prime} \alpha}(\log z)^{m^{\prime}}$ if either $k+l \alpha<k^{\prime}+l^{\prime} \alpha$ or $k+l \alpha=k^{\prime}+l^{\prime} \alpha$ and $m>m^{\prime}$. Arranged in this order, these products of powers of $z$ and $\log z$ form a sequence of functions $\chi_{n}$. The coefficients in these expansions are complex constants, some of which may be zero.

From Theorem 1 an asymptotic expansion for the inverse function $F^{-1}(z)$, which maps the domain $D$ onto a portion of the upper half plane, can be obtained easily by replacing the asymptotic expansions by finite developments with error terms and proceeding as usual in the inversion of functions. The result obtained is stated in the following theorem.

THEOREM 2. If $\alpha$ is irrational, then for $z \rightarrow 0$ in any finite sector the inverse of $F(z)$,

$$
F^{-1}(z) \sim \sum B_{k l} z^{k+l / \alpha}
$$

where $k$ and $l$ run over integers, $k \geq 0, l \geqq 1$; and $B_{01} \neq 0$. If $\alpha=p / q$, a fraction reduced to lowest terms, then for $z \rightarrow 0$ in any finite sector

$$
F^{-1}(z) \sim \sum B_{k l m} z^{k+l / \alpha}(\log z)^{m}
$$

where $k, l$ and $m$ run over integers for which $k \geqq 0,1 \leqq l \leqq p, 0 \leqq m \leqq k / q$; and $B_{010} \neq 0$.

There is another way to state Theorems 1 and 2 in the case of rational $\alpha$. We can write

$$
F(z) \sim z^{1 / \alpha} M_{1}\left(z, z^{1 / \alpha}, z^{q} \log z\right)
$$

and

$$
F^{-1}(z) \sim z^{\alpha} M_{2}\left(z, z^{\alpha}, z^{p} \log z\right)
$$

where $M_{1}$ and $M_{2}$ are triple power series in their three arguments. In the case $\alpha=1$ the triple power series reduces to a double series in $z$ and $z \log z$ as found by Lewy [4].

Observe that the function $F(z)$, defined originally for $0 \leqq \arg z \leqq \pi$, can be extended by the reflection principle across both the positive $x$-axis and the negative $x$-axis since the curves $\Gamma_{1}$ and $\Gamma_{2}$ are analytic curves. The images of $\Gamma_{1}$ and $\Gamma_{2}^{\prime}$ in such reflections are again analytic curves. Hence $F(z)$ can again be extended by reflection, and in fact can be continued near the origin onto the entire logarithmic Riemann surface with 
branch point at the origin. The function $F(z)$ is regular for $|z|$ sufficiently small, say, $0<|z|<\rho$, on any sheet of this Riemann surface ; but, generally speaking, $\rho$ depends on the sheet of the surface.

4. Extension of developments to larger sectors. If the asymptotic expansions of Theorems 1 and 2 hold for $z \rightarrow 0$ in $0 \leqq \arg z \leqq \pi$, they hold for $z \rightarrow 0$ in any finite sector $\theta_{1} \leqq \arg z \leqq \theta_{2}$. Suppose, indeed, that for given $r>0, F(z)$ has a finite development of the form

$$
F(z)=\sum A_{k l m} z^{k+l \alpha}(\log z)^{m}+o\left(z^{r}\right)
$$

as $z \rightarrow 0,0 \leqq \arg z \leqq \pi$, where the sum is extended over integers $k, l$, and $m$ such that $k+l \alpha \leqq r, k \geqq 0, l \geqq 1$; and $0 \leqq m \leqq k / p$ when $\alpha=p / q, m=0$ when $\alpha$ is irrational. Then the same development is valid for $z \rightarrow 0$ with $-\pi \leqq \arg z \leqq 0$. To see this let $\zeta^{*}$ be the image of $\zeta$ in an analytic reflection on the curve $\Gamma_{1}$. Then $\bar{\zeta}^{*}$, the complex conjugate of $\zeta^{*}$, is an analytic function of $\zeta$, say $\Phi(\zeta)$, which is regular for $|\zeta|$ sufficiently small. By the reflection principle, since $F(z)$ takes the positive real axis, $\arg z=0$, into the analytic curve $\Gamma_{1}$, we have

$$
F(\bar{z})=(F(z))^{*}=\overline{\Phi(F(z))}
$$

for $0 \leq \arg z \leq \pi$. Observe that this formula continues $F(z)$ for $|z|$ sufficiently small into the sector $-\pi \leqq \arg z \leqq \pi$. Since $\Phi(\zeta)$ is regular for $|\zeta|$ sufficiently small and $\Phi(0)=0$, we have

$$
\Phi(\zeta)=\sum_{n=1}^{[r / \alpha]} c \zeta^{n}+o\left(\zeta^{r / \alpha}\right)
$$

for $z \rightarrow 0$. Then with

$$
\zeta=F(z)=z^{\alpha}\left[\sum c z^{k+l \alpha}(\log z)^{m}+o\left(z^{r-\alpha}\right)\right], \quad(k \geqq 0, l \geqq 0, k+l \alpha \leq r-\alpha)
$$

we have by (4.1) for $z \rightarrow 0,0 \leqq \arg z \leqq \pi$,

$$
\zeta^{n}=z^{n \alpha}\left[\sum c z^{k+l \alpha}(\log z)^{m}+o\left(z^{r-n \alpha}\right)\right]
$$

where $k \geqq 0, l \geqq 0, k+l \alpha \leqq r-n \alpha ; m$ is limited as before. Also

$$
o\left(\zeta^{r / \alpha}\right)=o\left(\left(O\left(z^{\alpha}\right)\right)^{r / \alpha}\right)=o\left(z^{r}\right)
$$

as $z \rightarrow 0$. Consequently for $z \rightarrow 0,0 \leqq \arg z \leqq \pi$,

$$
F(\bar{z})=\overline{\Phi(F(z))}=\sum c \bar{z}^{k+l \alpha}(\log \bar{z})^{m}+o\left(\bar{z}^{r}\right)
$$

where $k, l$, and $m$ are restricted in the same way as in (4.1). But this means that $F(z)$ has a development of the same type as (4.1) for $-\pi \leqq \arg z \leqq 0$. This new development must coincide with that given by (4.1) since both hold for $z \rightarrow 0$ with $\arg z=0$. 
In the same way we can reflect across the line $\arg z=\pi$ and establish that (4.1) holds in the larger sector thus obtained. By induction we can prove that (4.1) holds in any finite sector $\theta_{1} \leqq \arg z \leqq \theta_{2}$. Thus we see that if Theorem 1 holds for $z \rightarrow 0$ in the sector $0 \leqq \arg z \leqq \pi$, it holds for $z \rightarrow 0$ in any finite sector.

5. Some lemmas. We now state some lemmas which will be used in the proof of Theorem 1. Lemmas 1 and 2 are special cases of Theorems 4.1 and 4.2 of [3]. The integrals are Lebesgue integrals extended over positive values of $t$. The range of $z$ considered is $0<|z|<A$, $-2 \pi \leqq \arg z \leqq 0$. We take the branch of the analytic function of $z$, $\log (1-z \mid t)$ which is real for $0<z<t, \arg z=0$.

Lemma 1. Let $A$ be a positive real number, $\mu$ a real number $>-1$, and $n$ a nonnegative integer ; and let

$$
\varphi(z)=\int_{0}^{A} t^{\mu}(\log t)^{n} \log (1-z / t) d t .
$$

Then there is a power series $q(z)$, which converges for $|z|<A$, and a polynomial in $\log z, P(\log z)$, such that

$$
\varphi(z)+z^{\mu+1} P(\log z)+q(z) \text {. }
$$

If $\mu$ is an integer then, the polynomial $P$ is of degree $n+1$; and if $\mu$ is not an integer, it is of degree $n$.

LEMMA 2. Let $\beta(t)$ be a measurable function, bounded absolutely for $0<t<A$ and such that $\beta(t) \rightarrow 0$ as $t \rightarrow 0$ through positive real values. Let $\mu$ be a real number $>-1$ which is not an integer, and let

$$
\beta_{1}(z)=\int_{0}^{A} \beta(t) t^{\mu} \log (1-z / t) d t
$$

Then there is a power series $q(z)$ such that for $z \rightarrow 0$

$$
\beta_{1}(z)=q(z)+o\left(z^{\mu+1}\right) .
$$

LEMmA 3. Let $\mu$ be a real number. Let $\eta(z)$ be an analytic function, regular for $0<|z|<R, \theta_{1} \leqq \arg z \leqq \theta_{2}$ and such that $\eta(z)=o\left(z^{\mu}\right)$ for $z \rightarrow 0$ in the sector $\theta_{1} \leqq \arg z \leqq \theta_{2}$. Then the derivative

$$
\eta^{\prime}(z)=o\left(z^{\mu-1}\right)
$$

for $z \rightarrow 0$ in any sector in the interior of the sector $\theta_{1} \leqq \arg z \leqq \theta_{2}$.

A proof of Lemma 3 is obtained by estimating a Cauchy integral with path a circle about $z$ with radius $\delta|z|, \delta$ small. 
Lemma 4. Let $\lambda$ be a real number. Then for $z \rightarrow 0$ with $|\arg z|$ bounded, $\left|z^{-\lambda} F(z)\right|$ tends to zero if $\lambda<\alpha$ and tends to infinity if $\lambda>\alpha$.

A proof of Lemma 4 can be obtained by a study of the Poisson integral (see Gross [1, pp. 57-61]; the requirement that $z \rightarrow 0$ in an angle in the interior of $0 \leqq \arg z \leqq \pi$ can be eliminated by using the fact that $\Gamma_{1}$ and $\Gamma_{2}$ are analytic curves).

This lemma also follows from the theorem of Lichtenstein mentioned in the introduction.

6. Preliminary transformations. First we establish that the general case can be reduced to the special case in which the curve $\Gamma_{2}$ is an analytic curve tangent to the positive real axis and $\Gamma_{1}$ is a portion of the ray $\arg \zeta=-\pi \alpha$ in the $\zeta$ plane. Consider a function $\psi(\zeta)$, regular for $|\zeta|$ sufficiently small, for which $\psi(0)=0, \psi^{\prime}(0)=b \neq 0$, and which takes the analytic curve $\Gamma_{1}$ into the line $\arg \zeta=-\pi \alpha$. The function $\psi$ maps $\Gamma_{2}$ into an analytic curve tangent to the positive real axis. For the sake of simplicity of notation we carry through the proof in detail only for irrational $\alpha$.

Suppose that we know Theorem 1 in the special case in which $\Gamma_{1}$ is the line $\arg \zeta=-\pi \alpha$, then for $z \rightarrow 0$ we have

$$
\phi(F(z))=z^{\alpha}\left\{\sum C_{k l} z^{k+l \alpha}+o\left(z^{r}\right)\right\}
$$

where the sum is extended over integers $k$ and $l$ for which $k \geq 0, l \geq 0$, $k+l \alpha \leqq r$. In addition, we can suppose that $C_{00} \neq 0$. Then since the inverse

$$
\psi^{-1}(\zeta)=\frac{\zeta}{b}+\sum_{n=2}^{N} c \zeta^{n}+o\left(\zeta^{N}\right)
$$

as $\zeta \rightarrow 0$, we have

$$
\begin{aligned}
F(z)=\psi^{-1}\left(\psi(F(z))=\frac{1}{b}-z^{\alpha}\left\{\sum C_{l k} z^{k+l \alpha}\right.\right. & \left.+o\left(z^{r}\right)\right\}+z^{2 \alpha}\left\{\sum c z^{k+l \alpha}+o\left(z^{r}\right)\right\} \\
& +\cdots+z^{N \alpha}\left\{\sum c z^{k+l \alpha}+o\left(z^{r}\right)\right\}+o\left(z^{N \alpha}\right)
\end{aligned}
$$

for $z \rightarrow 0$. Hence by taking $N$ large enough, we obtain

$$
F(z)=z^{\alpha}\left\{\sum C_{k l}^{\prime} z^{k+l \alpha}+o\left(z^{r}\right)\right\}
$$

where $C_{00}^{\prime}=\frac{1}{b} C_{00} \neq 0$. All of the sums considered are extended over integers $k \geq 0, l \leqq 0, k+l \alpha \leqq r$. Thus we need consider only the special case in which $\Gamma_{1}$ is a portion of the line $\arg \zeta=-\pi \alpha$.

Now we make another preliminary transformation. Let $w=\zeta^{1 / \alpha}$, so that the line $\arg \zeta=-\pi \alpha$ goes into the negative real axis. The analytic curve $\Gamma_{2}$ goes into a curve $\Gamma^{\prime}$ tangent to the positive real axis. This 
new curve $\Gamma^{\prime}$ is not analytic at the origin; we will find it useful to have the equation of $\Gamma^{\prime}$.

Let $\zeta=\xi+i \eta$. The analytic curve $\Gamma_{2}$ is given by an equation with real $a_{j}$

$$
\eta=a_{2} \xi^{2}+a_{3} \xi^{3}+a_{4} \xi^{4}+\cdots
$$

for $\xi>0$, where the series is convergent for $\xi$ sufficiently small. Then on $\Gamma^{\prime}$ we have

$$
\begin{aligned}
w=\zeta^{1 / \alpha}=(\xi+i \eta)^{1 / \alpha} & =\xi^{1 / \alpha}\left(1+i a_{2} \xi+i a_{3} \xi^{2}+\cdots\right)^{1 / \alpha} \\
& =\xi^{1 / \alpha}\left(1+c \xi+c \xi^{2}+\cdots\right) .
\end{aligned}
$$

Separating real and imaginary parts, we have with $w=u+i v$

$$
\begin{aligned}
& u=\xi^{1 / \alpha}\left(1+c \xi^{2}+c \xi^{3}+\cdots\right) \\
& v=\xi^{1 / \alpha}\left(c \xi+c \xi^{2}+c \xi^{3}+\cdots\right) .
\end{aligned}
$$

Consequently,

$$
u^{\alpha}=\xi+c \xi^{2}+c \xi^{3}+\cdots
$$

and thus

$$
\xi=u^{\alpha}+c u^{2 \alpha}+c u^{3 \alpha}+\cdots .
$$

Hence we obtain finally that the curve $\Gamma^{\prime}$ is given by an equation of the form

$$
v=u \sum_{k=1}^{\infty} b_{k} u^{k \alpha}
$$

for $u \leq 0$, where the series converges for $u$ sufficiently small.

7. Obtaining the asymptotic expansion. Let $D^{\prime}$ be the image of $D$ under the transformation $w=\zeta^{1 / \alpha}$; we can now assume that near the origin $D^{\prime}$ is bounded by the negative real axis and the curve $\Gamma^{\prime}$ given by the equation (6.1). We consider the function $w=G(z)=(F(-z))^{1 / a}$ which is a univalent conformal mapping of a semi-neighborhood $y<0$ of the $z=x+i y$ plane into the domain $D^{\prime}$ of the $w=u+i v$ plane. Observe that $G(0)=0$, a portion $-A \leqq x \leqq 0$ of the negative $x$-axis is mapped into a portion of the negative $u$-axis, and a portion $0 \leqq x \leqq A$ of the positive $x$-axis goes into $\Gamma^{\prime}$.

We will need an estimate for $G(z)$ and its derivative $G^{\prime}(z)$. By Lemma 4 we have for $z \rightarrow 0,|\arg z|$ bounded

$$
G(z)=[F(-z)]^{1 / \alpha}=\left[o\left(z^{\lambda}\right)\right]^{1 / \alpha}
$$

for any $\lambda<\alpha$. Hence for any $\varepsilon>0$ 


$$
G(z)=o\left(z^{1-\varepsilon}\right)
$$

as $z \rightarrow 0$ with $|\arg z|$ bounded. Using Lemma 3 we conclude further that for $z \rightarrow 0$

$$
G^{\prime}(z)=o\left(z^{-\varepsilon}\right) .
$$

Now we construct a certain function $H(z)$ which differs from $G(z)$ by a single-valued function. Observe that the function

$$
G(z)=u(x, y)+i v(x, y)
$$

can be continued across the negative real axis, $\arg z=-\pi$, by the reflection principle. In particular, we have for $\arg z=0$

$$
\begin{aligned}
G(z)-\left(G z e^{-2 \pi i}\right) & =u(z, 0)+i v(z, 0)-[u(z, 0)-i v(z, 0)] \\
& =2 i v(z, 0) .
\end{aligned}
$$

Consider for $-2 \pi \leqq \arg z \leqq 0$ the analytic function

$$
H(z)=\frac{1}{\pi} \int_{0}^{4} \frac{\partial v(t, 0)}{\partial t} \log (1-z / t) d t
$$

where the integral is extended over positive real values and the branch of $\log (1-z / t)$ considered is the one which is real for $0<z<t, \arg z=0$. That the integral converges follows from the estimate (7.2).

For $\arg z=0$ we have

$$
H(z)-H\left(z e^{-2 \pi i}\right)=\frac{2 \pi i}{\pi} \int_{0}^{z} \frac{\partial v(t, 0)}{\partial t} d t=2 i v(z, 0)
$$

since

$$
\log (1-z / t)-\log \left(1-z e^{-2 \pi i} / t\right)= \begin{cases}2 \pi i & \text { for } t<z, \\ 0 & \text { for } t>z .\end{cases}
$$

Thus the difference $p(z)=G(z)-H(z)$ satisfies the condition $p(z)=p\left(z e^{-2 \pi i}\right)$ for $\arg z=0$. Furthermore $p(z)$ is regular for $0<|z|<A,-2 \pi<\arg z<0$; it is continuous as $z$ approaches a point of the positive real axis for $\arg z=0$ or $\arg z=-2 \pi$, and it is bounded for $z \rightarrow 0$. Hence by Riemann's theorem on removable singularities $p(z)$ is equal to a power series convergent for $|z|<A$.

From (6.1) and (7.3) we conclude that for $-2 \pi \leqq \arg z \leqq 0$

$$
G(z)=\frac{1}{\pi} \int_{0}^{A}\left\{\begin{array}{c}
\partial u(t, 0) \\
\partial t
\end{array} \sum_{n=1}^{\infty} b_{n}(1+n \alpha) u^{n \alpha}\right\} \log (1-z / t) d t+p(z)
$$


where $p(z)$ is a power series with constant term equal to zero.

By (7.1) and (7.2) we have $\frac{\partial u(t, 0)}{\partial t}=o\left(t^{-\varepsilon}\right)$ and $u^{\alpha}=o\left(t^{(1-\varepsilon) \alpha}\right)$ for $t \rightarrow 0, \varepsilon$ an arbitrary positive number. Hence for $t \rightarrow 0$

$$
\frac{\partial u(t, 0)}{\partial t} \sum_{n=1}^{\infty} b_{n}(1+n \alpha) u^{n \alpha}=o\left(t^{(1+\alpha)(1-\varepsilon)-1}\right) .
$$

Inserting this estimate in (7.4) and applying Lemma 2, we obtain for $z \rightarrow 0,-2 \pi \leqq \arg z \leqq 0$

$$
G(z)=a z+z^{2} q(z)+o\left(z^{(1+\alpha)(1-\varepsilon)}\right)
$$

where $q(z)$ is a power series in $z$ which converges for $|z|$ sufficiently small. We conclude that $a \neq 0$ by applying Lemma 4 with $\lambda$ slightly larger than $\alpha$. Knowing this, we can conclude further that $a$ is positive from the fact that $G(z)$ maps the positive real axis into $\Gamma^{\prime}$, a curve which at the origin makes an angle of $\pi$ with the negative real axis. Since $G(z)=[F(-z)]^{1 / \alpha}$ the result of $\S 4$ shows that the estimate (7.5) holds for $z \rightarrow 0$ in any finite sector.

Now we prove Theorem 1 by induction. We consider first the case in which $\alpha$ is irrational. We shall prove that there are constants $a_{k l}$ such that for every integer $N$,

$$
G(z)=\sum_{k+l \alpha \leqq N a} a_{k l} z^{k+l \alpha}+o\left(z^{N \alpha}\right), \quad k \geqq 1, l \geqq 0
$$

as $z \rightarrow 0$ with $|\arg z|$ bounded. We begin by noting that $G(z)$ has such a development for $N=N_{0}$ where $N_{0}$ is the integer for which $\frac{1}{\alpha} \leqq N_{0}<1$ $+\frac{1}{\alpha}$. This follows directly from (7.5) since for $\varepsilon$ sufficiently small $(1+\alpha)(1-\varepsilon) \geqq N_{0} \alpha$ and hence $o\left(z^{(1+\alpha)(1-\varepsilon)}=o\left(z^{N}{ }^{\alpha}\right)\right.$. Consequently, to prove (7.6) by induction it will be sufficient to show that if $G(z)$ has a development of the type (7.6) with an error term $0\left(z^{N \alpha}\right)$, then $G(z)$ has such a development with an error term $o\left(z^{(N+1) \alpha}\right)$. In proving (7.6) by induction we will simultaneously obtatin a proof of Theorem 1 by using the fact that $F(z)=[G(-z)]^{\alpha}$.

By the induction hypothesis we have

$$
u(t, 0)=\sum_{k+l \alpha \leqq N \alpha} \Re\left\{a_{k l}\right\} t^{k+l \alpha}+o\left(t^{N \alpha}\right), \quad(k \geq 1, l \geq 0),
$$

and thus since $a_{10}=a>0$

$$
u(t, 0)=a t\left\{1+\sum c t^{k+l \alpha}+o\left(t^{N \alpha-1}\right)\right\}
$$

where the sum is over $k \geq 0, l \geqq 0$, for which $(k, l) \neq(0,0)$ and $k+l \alpha$ $\leqq N \alpha-1$. Using the binomial theorem, we find 


$$
u^{n \alpha}=a^{n \alpha} t^{n \alpha}\left\{\sum_{k+l \alpha \leqq N \alpha-1} c t^{k+l \alpha}+o\left(t^{N \alpha-1}\right)\right\}, \quad(k \geqq 0, l \geqq 0) .
$$

Moreover, by Lemma 3 and the induction hypothesis we have for $t \rightarrow 0$

$$
\frac{\partial u(t, 0)}{\partial t}=\Re G^{\prime}(t)=\sum_{k+l \alpha \leqq N \alpha-1}(k+1+l \alpha) \Re\left\{a_{k+1, l}\right\} t^{k+l \alpha}+o\left(t^{N \alpha-1}\right)
$$

where $k \geqq 0, l \geqq 0$. Inserting these estimates in (7.4), we obtain

$$
G(z)=p(z)+\int_{0}^{A}\left\{\sum c t^{k+l \alpha}+o\left(t^{(N+1) \alpha-1}\right)\right\} \log (1-z / t) d t
$$

where the sum is over integers $k \geqq 0, l \geqq 1$, for which $k+l \alpha \leqq(N+1) \alpha-1$. Now we apply Lemmas 1 and 2 , observing that since $l \geq 1$ and $\alpha$ is irrational, $k+l \alpha$ cannot be an integer. We find for $z \rightarrow 0,-2 \pi \leqq \arg z \leqq 0$,

$$
G(z)=\sum_{k+l \alpha \leqq(N+1) \alpha} a_{k l} z^{k+l \alpha}+o\left(z^{(N+1) \alpha}\right), \quad(k \geq 1, \quad l \geq 0) .
$$

When $k$ and $l$ are integers for which $k+l \alpha \leqq N \alpha$, the coefficient $a_{k l}$ must, of ceurse, be the same as that appearing in the development with error term $o\left(z^{N a}\right)$.

We wish to prove that (7.7) holds for $z \rightarrow 0$ in any finite sector. We note that for $z \rightarrow 0,0 \leqq \arg z \leqq 2 \pi$

$$
F(z)=[G(-z)]^{\alpha}=a^{\alpha}(-z)^{\alpha}\left\{1+\sum c(-z)^{k+l \alpha}+o\left(z^{(N+1) \alpha-1}\right)\right\}^{\alpha}
$$

where the sum is over $k \geq 0, l \geq 0$, which for $(k, l) \neq(0,0)$ and $k+l \alpha$ $\leqq(N+1) \alpha-1$. Hence by the binomial theorem

$$
F(z)=\sum A_{k i} z^{k+l \alpha}+o\left(z^{(N+2) \alpha-1}\right)
$$

where the sum is extended over $k \geqq 0, l \geqq 1$, for which $k+l \alpha \leqq(N+2) \alpha-1$. Note further that $A_{01} \neq 0$. We have proved (7.8) for $z \rightarrow 0$ with $0 \leqq \arg z$ $\leqq 2 \pi$, but by the result of $\S 4$ this formula must hold for $z \rightarrow 0$ in any finite sector. Consequently, from (7.8) by using the binomial theorem we can obtain (7.7) for $z \rightarrow 0$ in any finite sector. Thus $G(z)$ has a development with error term $o\left(z^{(N+1) \alpha}\right)$. Hence by induction (7.6) and also (7.8) hold for all $N$. This proves Theorem 1 for irrational $\alpha$.

Now we prove Theorem 1 for $\alpha=p / q$, a fraction reduced to lowest terms. Let $\gamma$ be a positive irrational number less than $\alpha$. We shall prove that there are constants $a_{k l m}$ such that for every integer $N$, as $z \rightarrow 0$, in a finite sector

$$
G(z)=\sum_{k+l \alpha \leqq N \gamma} a_{k l m} z^{k+l \alpha}(\log z)^{m}+o\left(z^{N \gamma}\right)
$$

where $k \geq 1,0 \leqq l \leqq q-1$, and $0 \leqq m \leqq \frac{k-1}{p}$. We begin by noting that $G(z)$ has such a development for $N=N_{0}$ where $N_{0}$ is the integer for 
which $\frac{1}{\gamma} \leqq N_{0}<1+\frac{1}{\gamma}$, as can be seen directly from (7.5). Consequently, to prove (7.9) by induction it will be sufficient to show that if $G(z)$ has a development of the above type with error term $o\left(z^{N \gamma}\right)$, then it has such a development with error term $o\left(z^{(N+1) \gamma}\right)$.

By the induction hypothesis we have for positive $t \rightarrow 0$

$$
u(t, 0)=\sum_{k+l \alpha \leqq N \gamma} \Re\left\{a_{k l m}\right\} t^{k+l \alpha}(\log t)^{m}+o\left(t^{N \gamma}\right)
$$

where $k \geqq 1,0 \leqq l \leqq q-1, \quad 0 \leqq m \leqq \frac{k-1}{p}$. Since $a_{100}=a \neq 0$, we have

$$
u(t, 0)=a t\left\{1+\sum c t^{k+l \alpha}(\log t)^{m}+o\left(t^{N \gamma-1}\right)\right\}
$$

where the sum is over integers for which

$$
k \geqq 0 ; 0 \leqq l \leqq q-1 ; 0 \leqq m \leqq k / p ; \quad l o+l \alpha \leqq N \gamma-1 .
$$

Using the binomial theorem, we obtain

$$
u^{n \alpha}=a^{n \alpha} t^{n \alpha}\left\{\sum c t^{k+l \alpha}(\log t)^{m}+o\left(t^{N \gamma-1}\right)\right\}
$$

where $k, l$, and $m$ are restricted by the conditions (7.10). Moreover, by Lemma 3 and the induction hypothesis we have

$$
\frac{\partial u(t, 0)}{\partial t}=\sum c t^{k+l \alpha}(\log t)^{m}+o\left(t^{N \gamma-1}\right)
$$

where again $k, l$, and $m$ are restricted by the conditions (7.10).

Inserting these estimates in (7.4) we have, since

$$
o\left(t^{N \gamma+\alpha-1}\right)=o\left(t^{(N+1) \gamma-1}\right),
$$

the formula

$$
G(z)=p(z)+\int_{0}^{A}\left\{\sum c t^{k+l \alpha}+o\left(t^{(N+1) \gamma-1}\right)\right\} \log (1-z / t) d t .
$$

The sum in the integrand is extended over integers $k, l$, and $m$ for which

$$
k \geqq 0 ; \quad 1 \leqq l \leqq q ; \quad 0 \leqq m \leqq k / p ; \quad k+l \alpha \leqq(N+1) \gamma-1 .
$$

Now we apply Lemmas 1 and 2 to obtain a better development for $G(z)$. Note that $k+l \alpha=k+l p / q$ cannot be an integer unless $l=q$. Consequently terms of the form

$$
c t^{k+l \alpha}(\log t)^{m}
$$

in the integrand, with $l \neq q$, produce besides a power series only terms of the form 


$$
c z^{k+1+l \alpha}(\log z)^{m^{\prime}}
$$

with

$$
0 \leqq m^{\prime} \leqq m \leqq \frac{(k+1)-1}{p}
$$

in the development for $G(z)$. On the other hand, when $l=q$ they produce besides a power series only terms of the form

$$
c z^{k+1+l \alpha}(\log z)^{m^{\prime}}=c z^{k+1+1}(\log z)^{m^{\prime}}
$$

with

$$
0 \leqq m^{\prime} \leqq m+1 \leqq \frac{k}{p}+1=\frac{(k+p+1)-1}{p}
$$

In applying Lemma 2 we observe that $(N+1) \gamma-1$ is not an integer because $\gamma$ is irrational. Hence we conclude that for $z \rightarrow 0,-2 \pi \leqq \arg z \leqq 0$,

$$
G(z)=\sum_{k+l \alpha \lesssim(N+1) \gamma} a_{k l m} z^{k+l \alpha}(\log z)^{m}+o\left(z^{(N+1) \gamma}\right)
$$

where $k \geqq 1,0 \leqq l \leqq q-1$, and $0 \leqq m \leqq \frac{k-1}{p}$.

As in the case of irrational $\alpha$ we obtain from this the result

$$
F(z)=\sum A_{k l m} z^{k+l \alpha}(\log z)^{m}+o\left(z^{(N+1) \gamma+\alpha-1}\right),
$$

where the sum is over integers $k, l$, and $m$ for which

$$
k \geqq 0, \quad 1 \leqq l \leqq q, \quad 0 \leqq m \leqq k l p ; \quad k+l \alpha \leqq(N+1) \gamma+\alpha-1 .
$$

By (7.11) this result holds for $z \rightarrow 0$ with $0 \leqq \arg z \leqq 2 \pi$, but by the result of $\S 4$, it must hold for $z \rightarrow 0$ in any finite sector. From this we then obtain (7.11) for $z \rightarrow 0$ in any finite sector. Hence $G(z)$ has a development with error term $o\left(z^{(N+1) \gamma}\right)$. Thus by induction (7.9) and also (7.12) hold for all $N$. This completes the proof of Theorem 1.

We note finally that by Lemma 3 derivatives of $F(z)$ and $F^{-1}(z)$ of arbitrary order have asymptotic expansions which can be obtained by differentiating the expansion for $F(z)$ and $F^{-1}(z)$ termwise and then rearranging the terms in the new series in an appropriate order.

\section{REFERENCES}

1. W. Gross, Zum Verhalten der konformen Abbildung am Rande, Math. Z. 3 (1919), 43-64.

2. O. D. Kellogg, Harmonic functions and Green's integral, Trans. Amer. Math. Soc., 13 (1912), 109-132. 
3. R. S. Lehman, Dovelopments in the neighborhood of the beach of surface waves over an inclined bottom, Communications on Pure and Applied Mathematics, 7, No. 3, (1954), 393-439.

4. H. Lewy, Developments at the confluence of analytic boundary conditions, University of California Publications in Mathematics, 1, No. 7 (1950), 247-280; also Proceedings of the International Congress of Mathematics, (1950), 601-605.

5. L. Lichtenstein, Über die konforme Abbildung ebener analytischer Gebiete mit Ecken, J. Reine Angew. Math., 140 (1911), 100-119.

6. S. Warschawski, Úber das Verhalten der Ableitung der Abbildungsfunktion bei konformer Abbildung, Math., 35 (1932), 321-456.

7. ___ On a theorem of L. Lichtenstein, Pacific J. Math., 5 (1955), 835-840.

STANFORD UNIVERSITY 
